\title{
Effect of solvent-induced structural modifications on optical properties of CdS nanoparticles
}

\author{
PUJA CHAWLA*, GEETA SHARMA, S P LOCHAB ${ }^{\dagger}$ and NAFA SINGH \\ Department of Physics, Kurukshetra University, Kurukshetra 136 119, India \\ ${ }^{\dagger}$ Inter University Accelerator Centre, Aruna Asaf Ali Marg, New Delhi 110 067, India
}

MS received 13 April 2009; revised 7 June 2009

\begin{abstract}
We have investigated the effects of solvent used during synthesis on structural and optical properties of CdS quantum dots. Different methods of synthesis for the production of CdS quantum dots are presented. These are: (a) wet chemical co-precipitation in non-aqueous medium (i.e. methanol); (b) wet chemical co-precipitation in aqueous medium (deionized water) and (c) solid state reaction. It is demonstrated that the use of methanol as solvent leads to a strong enhancement of PL intensity of CdS quantum dots for use in optoelectronic devices. These products were characterized by X-ray powder diffraction (XRD), high resolution transmission electron microscopy (HRTEM) and scanning electron microscopy (SEM). The change in bandgap with size-quantization was investigated by UV-VIS absorption spectroscopy. CdS nanocrystals prepared in non-aqueous medium have narrow size distribution than those prepared in aqueous medium and solid state reaction. Phase transformation of $\mathrm{CdS}$ nanocrystals from a cubic to hexagonal structure was observed in methanol solution. The formation of $\mathrm{CdS} / \mathrm{Cd}(\mathrm{OH})_{2}$ nanostructure was also confirmed using X-ray diffraction pattern. This suggests that the strong enhancement of the PL intensity may have originated from the remarkable reduction of non-radiative recombination process, due to surface defects of quantum dots. The red shift of the Raman peaks compared to that for bulk CdS may be attributed to optical phonon confinement.
\end{abstract}

Keywords. CdS; nanoparticles; photoluminescence; red shift; Raman.

\section{Introduction}

Efficiently luminescing nanocrystalline semiconductors form an important class of luminescent materials (Alivisatos 1996a). Group II-VI semiconducting materials have attracted much attention as good phosphors that has been studied since 1971 (Lehmann and Ryan 1971; Lehmann et al 1972), with potential use in cathode ray tubes (Marwaha et al 1979), TV screens, fluorescent screens, fluorescence lamps and thermoluminescence dosimetry (Marwaha et al 1980, 1981). By controlling the crystalline size one can tailor the optical properties of the nanocrystallites. The stability, tunability and higher efficiency of the luminescent nanocrystallites may replace all existing bulk phosphors in the future. For instance, it has been shown that smaller the particle size, the higher the screen resolution and lower the screen loading (Kumar et al 2006).

Materials on micrometric scale mostly exhibit physical properties that are the same as that of bulk form; however, materials on the nanometer scale may exhibit physical properties distinctly different from those of the bulk. For example, a bulk semiconductor material becomes an

\footnotetext{
*Author for correspondence (ranu.puja@gmail.com)
}

insulator when the characteristic dimension is sufficiently small. There are two reasons for the change of electronic states in nanosized particles-one reason is the quantum size effect (Alivisatos 1996a), which is a consequence of the strong reduction of allowed states in the small particles resulting in an increase in the bandgap; the other reason is the much larger surface to volume ratio, which makes surface and interface effects dominant over volume effects. Moreover, from these nanophosphors we can achieve advanced optical properties when the scale of confinement approaches the excitonic Bohr radius $\left(r_{\mathrm{B}}\right)$, which sets the length scale for optical processes.

$\mathrm{CdS}$ is a wide bandgap semiconductor with $E_{\mathrm{g}} \approx 2.5 \mathrm{eV}$ (Zou et al 1999). It is used in photodetectors and for solar cell applications as $n$-type window layers in heterojunction devices (Danaher et al 1985). The optical properties of CdS have been extensively studied (Colvin et al 1994; Vossmeyer et al 1994; Nag et al 2008). Quantum size effects are quite pronounced because $\mathrm{CdS}$ has a rather large $r_{\mathrm{B}}(\approx 3 \mathrm{~nm})$ (Alivisatos 1996b). CdS quantum dots are attractive candidates for optoelectronic applications as it is possible to engineer the bandgap over a wide spectral range (visible to UV). Several synthetic routes have been used to produce II-VI nanocrystals i.e. flux method, solvothermal route, wet chemical co-precipitation method and solid state method (Nanda et al 1999; Patil and Wani 
2001; Wang et al 2003; Yu et al 2004; Kumar et al 2006; Sun et al 2006; Anila et al 2008; Orimi et al 2008). To obtain a clearer understanding of the relative importance of the solvent used during synthesis on the structural and optical properties of $\mathrm{CdS}$ nanoparticles, we have prepared $\mathrm{CdS}$ nanoparticles via different routes. Herein we describe and discuss the current use of chemical methods such as those based on solid state reaction, wet chemical co-precipitation method in both aqueous and non-aqueous medium to prepare $\mathrm{CdS}$ nanophosphors. In our experiments, deionized water and methanol were used as solvent. It is found that the solvent has a great influence on the phase structure, the product composition and the mean size of the CdS nanocrystallites. As a result a drastic change in photoluminescent properties was observed. Thus, the aim of our present study is to highlight various strategies for obtaining highly photoluminescent and water dispersed $\mathrm{CdS}$ nanocrystals for use in optoelectronic devices. The obtained nanophosphors hold great promise for future technological applications. The structural characterization of these materials have been carried out by XRD, TEM and SEM. We have then studied these materials using absorption, emission and Raman spectroscopies.

\section{Experimental}

$\mathrm{CdS}$ nanocrystals have been synthesized based on wet chemical co-precipitation in non-aqueous (methanol) or aqueous (deionized water) solvent and solid state reaction. All chemicals and solvents were of analytical grade and used as-received without further purification.

In a typical experiment using wet chemical coprecipitation method, a $0.0125 \mathrm{M}$ sulfide solution was prepared by dissolving $0.3002 \mathrm{~g}$ of $\mathrm{Na}_{2} \mathrm{~S} \cdot 9 \mathrm{H}_{2} \mathrm{O}$ and $0.11 \mathrm{ml}$ of thioglycerol in $100 \mathrm{ml}$ of methanol. A $0.025 \mathrm{M}$ $\mathrm{CdCl}_{2} \cdot \mathrm{H}_{2} \mathrm{O}$ solution was prepared by dissolving $0.5033 \mathrm{~g}$ of $\mathrm{CdCl}_{2} \cdot \mathrm{H}_{2} \mathrm{O}$ in $100 \mathrm{ml}$ of methanol. The organic capping agent (1-thioglycerol) was mixed with the solution to control the particle size. For the synthesis of $\mathrm{CdS}$ nanoparticles, $100 \mathrm{ml}$ of $\mathrm{CdCl}_{2}$ solution was placed in a three-necked round-bottom flask fitted with valves and a stopper. The solution was deoxygenated with nitrogen for $15 \mathrm{~min}$, followed by swift injection of $100 \mathrm{ml}$ of sulfide solution under vigorous stirring. The mixture was stirred for $3 \mathrm{~h}$ at room temperature. Particle growth occurred at this stage via Oswald ripening. Then the mixture was concentrated down to $\sim 50 \mathrm{ml}$ in volume by heating and tetrahydrofuran (THF) was added dropwise. The solid portion was collected by centrifugation and washed with deionized water and ethanol several times and dried in vacuum oven. The final products were the light yellow $\mathrm{CdS}$ nanoparticles. The same procedure was tried with different solvents i.e. deionized water, methanol and ethanol for preparing cadmium and sulfide solutions.
Thus yellow $\mathrm{CdS}$ nanoparticles in powder form were obtained using chemical co-precipitation technique.

In case of using solid state reaction, $5 \cdot 1495 \mathrm{~g} \mathrm{CdCl}_{2} \cdot \mathrm{H}_{2} \mathrm{O}$ and $1.9963 \mathrm{~g} \mathrm{Na} 2 \mathrm{~S} \cdot x \mathrm{H}_{2} \mathrm{O}$ were taken and ground for 10 min each in a clean agate pestle-mortar. Then these were mixed together in a cleaned agate mortar and ground for further $40 \mathrm{~min}$ to get good homogeneity. The orange products were washed several times with distilled water and twice with ethanol to remove $\mathrm{NaCl}$ and unreacted precursors completely and then centrifuged. Finally, the products were dried for $8 \mathrm{~h}$ in vacuum oven at $60^{\circ} \mathrm{C}$. The orange products were then collected in clean and dry sample bottles.

The structural characterization of the samples was carried out by X-ray powder diffraction (XRD) using Bruker Advance D8 X-ray diffractometer using $\mathrm{CuK} \alpha$ radiation. The morphology and sizes of the products were determined by transmission electron microscopy (TEM) carried out using H-7500 (Hitachi Ltd Tokyo Japan) operated at $120 \mathrm{kV}$. Diluted nanoparticles suspended in acetone were introduced on a carbon coated copper grid, and were allowed to dry in air for conducting TEM studies. Field emission scanning electron microscopy (FESEM) along with energy dispersive spectroscopy (EDAX) were used to investigate the morphology and composition of as-prepared products. Photoluminescence was recorded with a FluoroMax-3 (Jobin-Yvon, Edison, NJ, USA) equipped with photomultiplier tube and a xenon lamp. The optical absorption spectra were recorded on an UV-VIS 2500PC spectrophotometer (Shimadzu Corp., Japan). Raman spectrometer (Jobin-Yvon model HR 800) was used to record Raman spectra.

\section{Results and discussion}

The diffraction patterns (figure 1) of CdS nanocrystals show features typical of nanodimensional particles. Figure 1(a) shows XRD pattern of the CdS samples prepared by solid state reaction. Figures $1(\mathrm{~b})$ and (c) show the XRD patterns of the CdS samples prepared by wet chemical co-precipitation, using $\mathrm{CdCl}_{2} \cdot \mathrm{H}_{2} \mathrm{O}$ as $\mathrm{Cd}^{2+}$ source and $\mathrm{Na}_{2} \mathrm{~S} \cdot 9 \mathrm{H}_{2} \mathrm{O}$ as $\mathrm{S}^{2-}$ source in the solvents deionized water and methanol, respectively. All three peaks in figures 1 (a) and (b) at $26.8^{\circ}, 43.9^{\circ}$ and $52.0^{\circ}$, can be indexed to cubic phase $\beta$-CdS by comparison with data from JCPDS file no 10-454. The broadness of peaks clearly indicates that the dimensions of the CdS nanoparticles are very small. Employing Scherrer's equation (Kaeble et al 1967), the mean size of the CdS nanocrystallites synthesized by various methods were calculated and are shown in table 1. Furthermore, it is interesting to note that phase transformation of $\mathrm{CdS}$ nanocrystals from a cubic to hexagonal structure was observed when medium changed from aqueous to non-aqueous. Nevertheless, by comparing the three patterns of CdS nanocry- 
Table 1. Size of CdS samples using Debye Scherrer's relation.

\begin{tabular}{lcccc}
\hline Sample & $2 \theta(\mathrm{deg})$ & FWHM & Grain size $(\mathrm{nm})$ & Phase \\
\hline 1(a) Solid state reaction & 26.585 & $2 \cdot 2684$ & 3.5 & Cubic \\
1(b) Chemical co-precipitation in deionized water & 27.019 & $3 \cdot 1379$ & $2 \cdot 6$ & Cubic \\
1(c) Chemical co-precipitation in methanol & 26.896 & 4.5936 & $1 \cdot 7$ & Cubic + hexagonal \\
\hline
\end{tabular}

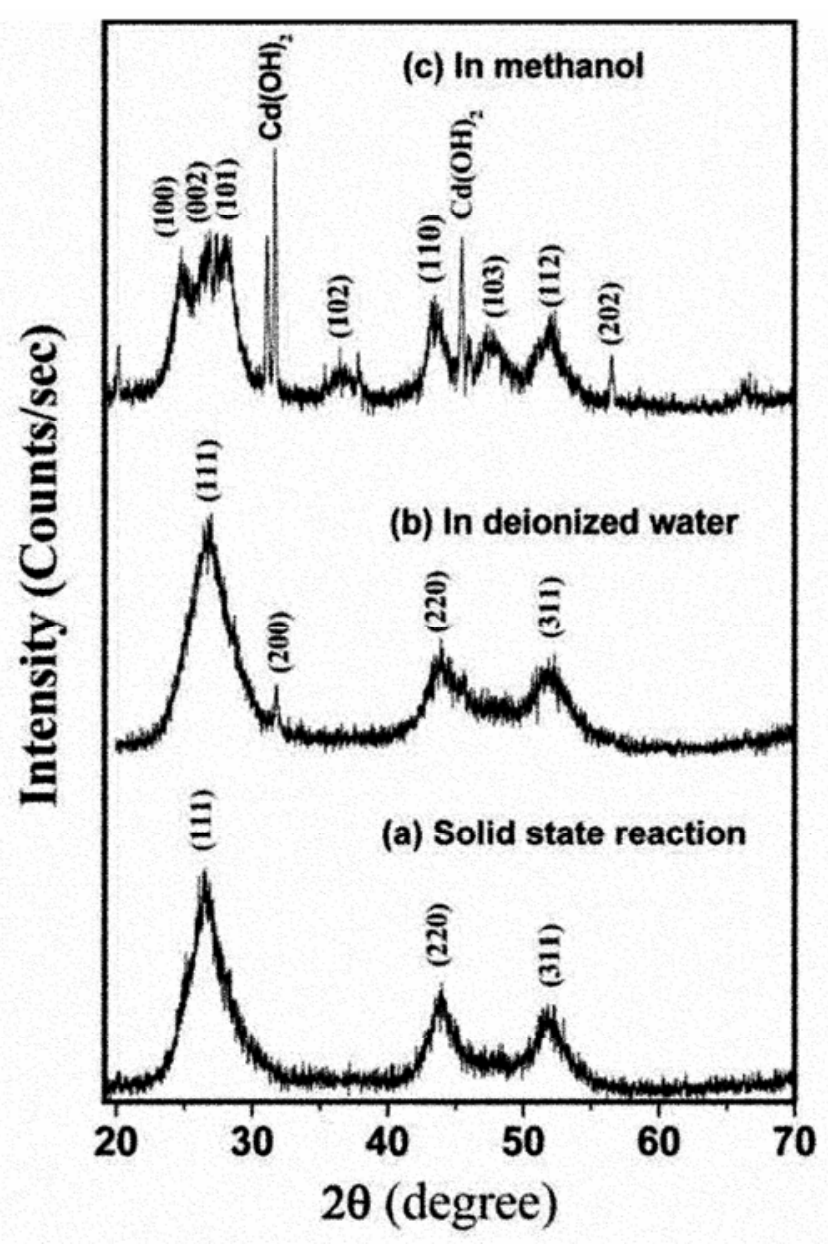

Figure 1. XRD patterns of three samples: (a) CdS nanocrystals prepared by solid state reaction, (b) CdS nanocrystals prepared in aqueous medium (deionized water) and (c) CdS nanocrystals prepared in non-aqueous medium (in methanol).

stals, we can conclude that in the third case (in methanol) the peaks (in the region $d \theta \approx 25^{\circ}-30^{\circ}$ ) related to the crystalline planes of the cadmium sulfide structure are observed. The (110), (103) and (112) planes of wurtzite CdS (Azad et al 2002) are well defined (see figure 1(c)). Whereas, in the first and second cases these planes appearing as broad peaks are not well defined. The appearance of these peaks (in figure 1(c)) indicates very small sizes of $\mathrm{CdS}$ nanocrystals and phase transformation. In figure $1(\mathrm{c})$ we can observe the peaks at $31.58^{\circ}$ and $45.61^{\circ}$ which indicate the presence of $\mathrm{Cd}(\mathrm{OH})_{2}$ phase along with $\mathrm{CdS}$ nanoparticles. Thus structure of $\mathrm{CdS}$ changed from zinc-blende to a mixture of zinc-blende and wurtzite (hexagonal); as synthesis medium changed to non-aqueous (methanol).

FESEM was performed to investigate the morphology of CdS nanocrystals. Figures 2(a)-(c) show the SEM images of as-synthesized $\mathrm{CdS}$ nanoparticles. All these images give almost spherical shape of nanoparticles. However, the nanoparticles obtained via wet chemical co-precipitation method in methanol (figure 1(c)) are well distributed homogenously.

The composition of as-prepared products were further confirmed by energy dispersive X-ray spectroscopy (EDAX), as shown in figures 2(a)-(c) along with SEM images. EDAX shows the presence of $\mathrm{Cd}, \mathrm{S}$ and $\mathrm{Cl}$ with different concentrations. The quantification calculation shows the atomic ratio of $\mathrm{Cd} / \mathrm{S}$ to be nearly $1: 1$. The presence of $\mathrm{Cl}$ may be due to $\mathrm{CdCl}_{2}$ used in the synthesis process. These results clearly reveal that the material shown in the above figures is exactly $\mathrm{CdS}$ having compositions consistent with the calculated ones.

A series of CdS nanocrystal samples were synthesized in deionized water, methanol, and solid state reaction. Figures 3(a)-(e) show TEM results of CdS nanocrystals synthesized using different methods. Figure 3(a) shows a typical TEM image of $\mathrm{CdS}$ nanocrystals synthesized using wet chemical co-precipitation in methanol, it can be seen that the $\mathrm{CdS}$ nanoparticles are well defined and nearly spherical with slight prolate deviations. The inserted SAED pattern shows the presence of diffraction rings which corresponds to the hexagonal wurtzite $\mathrm{CdS}$. Figure 3(b) shows high resolution electron micrograph (HRTEM) of an assembly of CdS nanoparticles. One can see well defined lattice fringes which demonstrate good crystallinity of the nanoparticles even at such a small size. The histogram for size distribution of CdS nanocrystals (figure 3(c)) shows crystal sizes ranging from $1-2.5 \mathrm{~nm}$. The average crystal size is $1.7 \pm 0.3 \mathrm{~nm}$.

Figure 3(d) shows TEM results of CdS nanocrystals synthesized by wet chemical co-precipitation in deionized water. The information derived from figure $3(\mathrm{~d})$ is similar to that from figure 3(a). One can see that the $\mathrm{CdS}$ nanocrystals synthesized in deionized water are also nearly spherical. The inserted SAED pattern shows the presence of diffraction rings which correspond to the cubic phase of $\mathrm{CdS}$. The average crystal size is $2.5 \mathrm{~nm}$. Figure 3(e) shows TEM results of CdS nanocrystals synthesized by solid state reaction. The TEM image indi- 

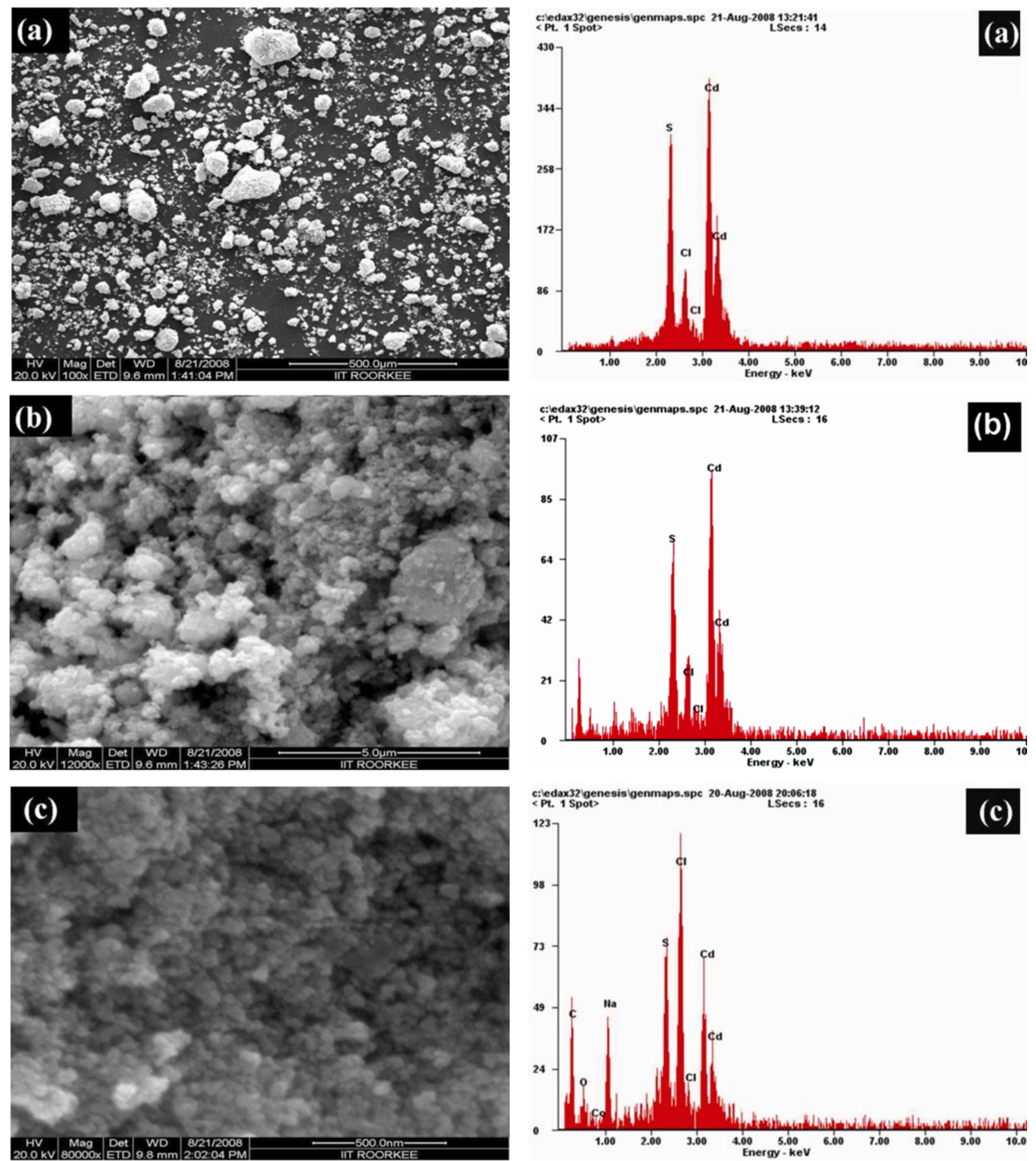

Figure 2. SEM and EDAX images of as-synthesized CdS nanoparticles using (a) solid state reaction, (b) chemical coprecipitation in aqueous medium and (c) chemical co-precipitation in methanol.

cates that the CdS nanoparticles are nearly spherical and agglomerated, with cubic phase confirmed using SAED pattern.
UV-VIS absorption spectra were recorded to investigate the quantum size effect of CdS nanocrystals. CdS is a typical direct bandgap semiconductor. When the size of 

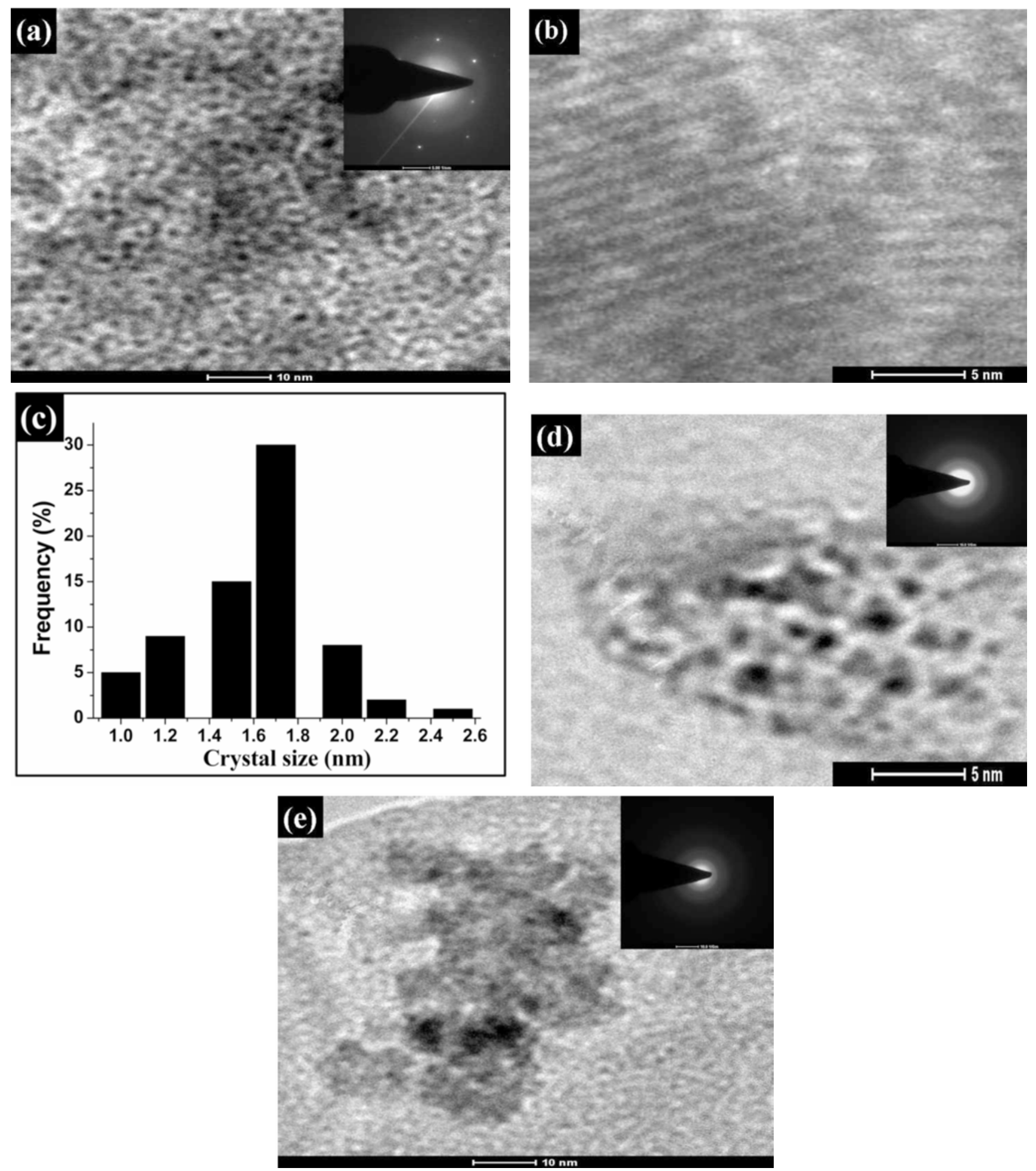

Figure 3. TEM results of CdS samples prepared by chemical co-precipitation reaction using methanol as solvent (a) TEM image and inserted SAED pattern, (b) HRTEM image, (c) histogram for crystal size distribution, (d) TEM image of CdS nanoparticles produced by chemical co-precipitation using deionized water as solvent and (e) TEM image of CdS samples prepared using solid state reaction.

CdS nanocrystals becomes smaller than the exciton radius, a remarkable quantum size effect leads to a size dependent increase of bandgap and a blueshift in the absorption maxima. Figure 4 shows UV-VIS absorption spectra of $\mathrm{CdS}$ nanocrystals obtained via different methods. The absorption onset in all these figures show a 
clear blue-shift, corresponding to a bandgap of $2.42 \mathrm{eV}$ for bulk $\mathrm{CdS}$. This blueshift in absorption is also a signature of nanostructure formation. The sharp absorption peak of $\mathrm{CdS}$ nanocrystals prepared via wet chemical co-precipitation in methanol implies that crystal size is smaller (i.e. $1.7 \mathrm{~nm}$ ) than the excitonic Böhr radius. For $\mathrm{CdS}$ synthesized in aqueous medium (i.e. deionized water) the absorption onset turns into a shoulder, implying that the crystal size distribution is defocussing due to growth of larger nanocrystals. This indicates that $\mathrm{CdS}$ nanocrystals prepared by wet chemical co-precipitation in methanol are smaller than those prepared in aqueous medium or solid state reaction. One can see clearly that the variation trend in absorption onset is in close agreement with quantum size effect.

The photoluminescence (PL) emissions from CdS nanoparticles synthesized by solid state reaction, wet chemical co-precipitation in aqueous medium and in nonaqueous medium (methanol) are shown in figures 5(a), 5(b) and 5(c), respectively. While the CdS nanoparticles synthesized by wet chemical co-precipitation in aqueous medium exhibit significant PL emission, the CdS nanoparticles synthesized by solid state reaction show very dim emission. Presumably the emission is weak due to the fact that most of the excited electron-hole pairs relax nonradiatively at surface states, therefore, PL emission of CdS nanocrystals produced by solid state method is very dim. Compared to the PL emissions shown in figure 5 (curves a,b), CdS nanocrystals synthesized by wet chemical co-precipitation in methanol (figure 5(c)) show much more intense $\approx 35$ times stronger PL emission. In contrast, Kim et al (2005) reported the activation of $\mathrm{CdS}$ nanocrystals originating from reverse micelle method. Hence, we tried such a surface modification to

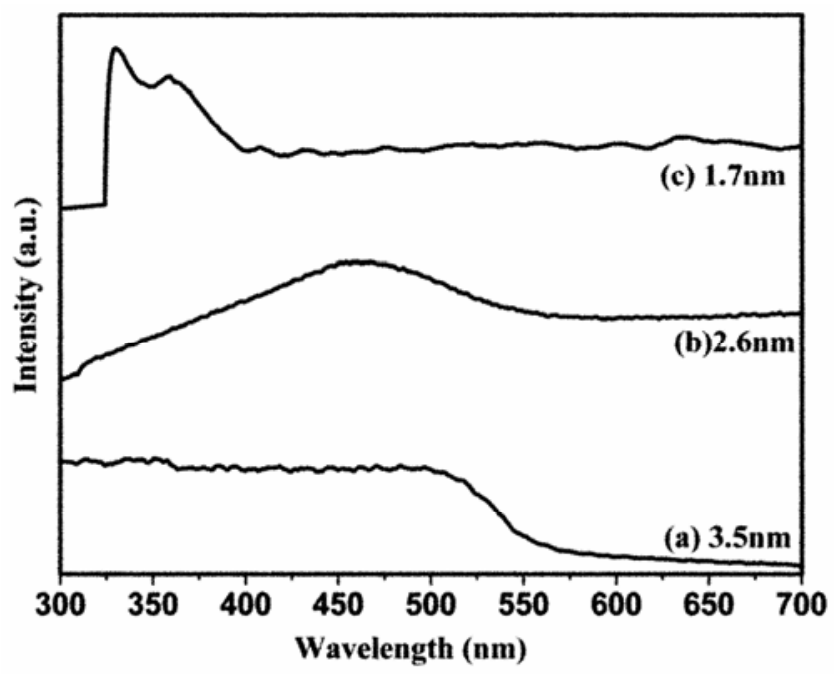

Figure 4. UV-VIS spectra of CdS nanoparticles prepared by (a) solid state reaction, (b) chemical co-precipitation in deionized water and (c) chemical co-precipitation in methanol. improve the PL properties of CdS quantum dots prepared by wet chemical co-precipitation reaction. Our results demonstrate that use of methanol as solvent in chemical co-precipitation reaction markedly improves the PL properties, which is due to the formation of $\mathrm{Cd}(\mathrm{OH})_{2}$ layer on the surface of CdS quantum dots. The formation of $\mathrm{Cd}(\mathrm{OH})_{2}$ layer on $\mathrm{CdS}$ quantum dots is also confirmed by XRD pattern (figure 1(c)), where the presence of peaks at $31.58^{\circ}$ and $45.61^{\circ}$ corresponds to $\mathrm{Cd}(\mathrm{OH})_{2}$ phase. This suggests that the formation of $\mathrm{Cd}(\mathrm{OH})_{2}$ results in removal of the CdS surface trap states, enhancing the PL intensity.

In PL spectra (figure 5), the broad PL emission with a large stokes shift, conventionally attributed to defects, is dominant. The broad emission peak at $550 \mathrm{~nm}$ (figures $5(a, b))$ are attributed to localized surface states in bandgap. While the emission at $640 \mathrm{~nm}$ (figure 5(c)) originates from internal defect emission, probably due to sulfur vacancies (Levy et al 1998; Sun et al 2000). The enhancement of PL intensity plus the lack of surfacerelated emission presumably results from suppression of nonradiative recombination due to reduced concentrations of surface states. These results demonstrate that although
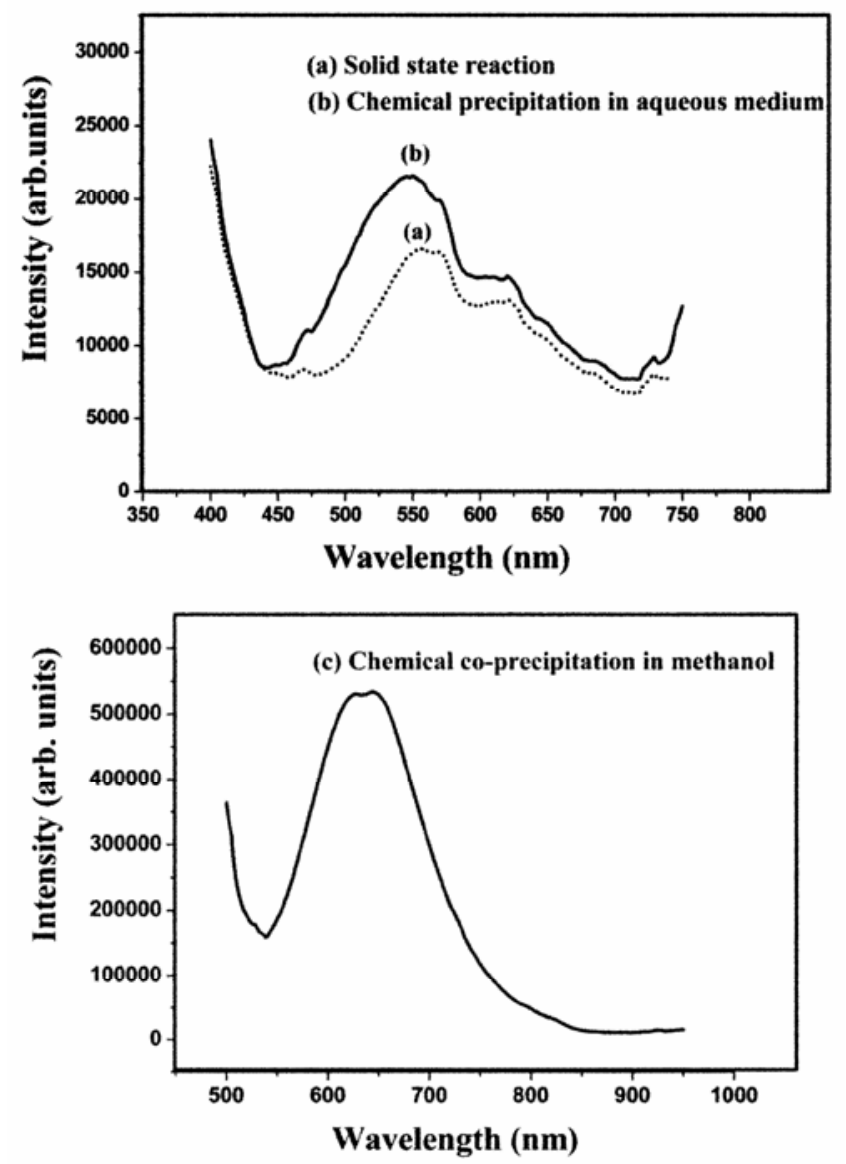

Figure 5. PL spectra of CdS nanoparticles synthesized by (a) solid state reaction, (b) chemical co-precipitation in aqueous medium and (c) chemical co-precipitation in methanol. 


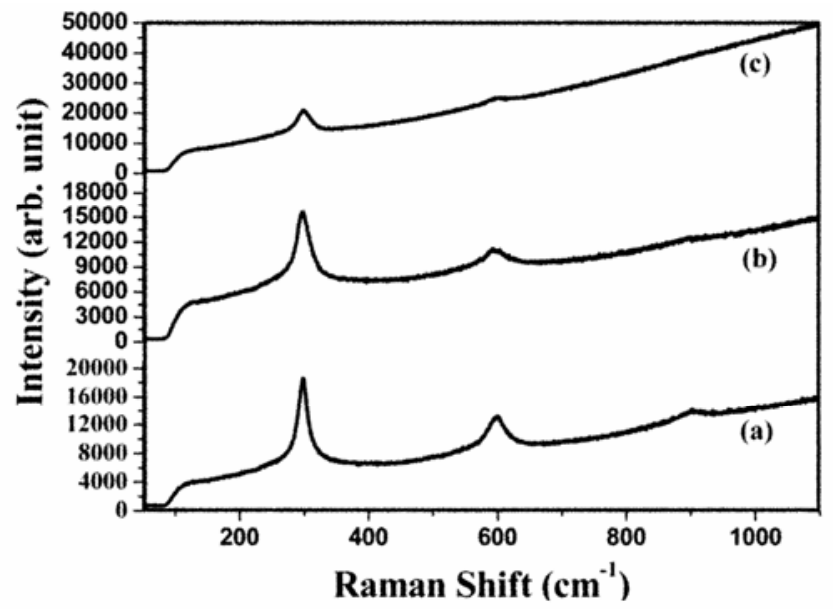

Figure 6. Raman spectra of CdS nanoparticles synthesized by (a) solid state reaction, (b) chemical co-precipitation method in aqueous medium and (c) chemical co-precipitation method in non-aqueous medium.

it is convenient to prepare CdS nanocrystals by solid state reaction and wet chemical co-precipitation in aqueous medium, very less PL intensity was observed. In contrast, the optical property was significantly improved for $\mathrm{CdS}$ nanocrystals prepared using methanol as solvent.

Raman spectra excited at $488 \mathrm{~nm}$ of CdS nanoparticles synthesized by various methods are displayed in figure 6 . The Raman spectra for as-prepared CdS nanoparticles contains peaks related to the longitudinal optical (LO) phonon and its overtones. The Raman-active 1LO phonon peak in bulk CdS was reported at $305 \mathrm{~cm}^{-1}$ (Tell et al 1966). In the present study, we observed Raman peaks for all these samples are red-shifted as compared to that of bulk. This red shift in comparison to the bulk CdS may be attributed to the effect of phonon confinement (Chuu and Dai 1992; Arora et al 2007).

\section{Conclusions}

We have investigated the effects of solvent used during synthesis on structural and optical properties of $\mathrm{CdS}$ quantum dots. The combination of XRD, TEM and UVVIS absorption measurements indicate that we have synthesized cadmium sulfide nanoparticles by various methods. CdS nanocrystals with controllable size and narrow size distribution have been prepared by solid state reaction and wet chemical co-precipitation in both aqueous and non-aqueous media. Phase transition from cubic to hexagonal structure was observed during the growth of $\mathrm{CdS}$ nanocrystals in methanol. The presence of $\mathrm{Cd}(\mathrm{OH})_{2}$ phase was also observed for $\mathrm{CdS}$ nanocrystals prepared in methanol as confirmed by XRD pattern. Strong photoluminescence was observed in CdS nanocrystals prepared in methanol. Noticeable shifts in absorption and PL spectra of $\mathrm{CdS}$ nanocrystals with change of solvents are accompanied with an improvement of their emission properties. The frequency of the CdS-Raman peaks, experimentally observed for the $\mathrm{CdS}$ nanocrystals, is somewhat red shifted than the bulk CdS LO phonon frequency and is attributed to phonon-confinement.

\section{References}

Alivisatos A P 1996a J. Phys. Chem. 10013226

Alivisatos A P 1996b Science 271933

Anila E I, Arvind A and Jayaraj M K 2008 Nanotechnology 19 145604

Arora A K, Rajalakshmi M, Ravindran T R and Subramanian V 2007 J. Raman Spectrosc. 38604

Azad M M, O'Brien P and Revaprasadu N 2002 Chem. Mater. 142004

Chuu D R and Dai C M 1992 Phys. Rev. B45 11805

Colvin V L, Schlamp M C and Alivisatos A P 1994 Nature 370 354

Danaher W I, Lyons L E and Morris G C 1985 Solar Energy Mater. 12137

Kaeble E F 1967 Handbook of X-rays (New York: McGrawHill)

Kim D, Miyamoto M, Mishima T and Nakayama M $2005 \mathrm{~J}$. Appl. Phys. 98083514

Kumar V, Singh N, Kumar R and Lochab S P 2006 J. Phys. Condens. Matter 185029

Lehmann W 1972 J. Lumin. 587

Lehmann W and Ryan F M 1971 J. Electrochem. Soc. 118477

Levy L, Ingert D, Feltin N and Pileni M P 1998 J. Cryst. Growth 184-185 377

Marwaha G L, Singh N and Mathur V K 1979 Mater. Res. Bull. 141489

Marwaha G L, Singh N and Mathur V K 1980 Radiat. Eff. 53 25

Marwaha G L, Singh N, Nagpal J S and Mathur V K 1981 Radiat. Eff. $\mathbf{5 5} 85$

Nag A, Sapra S, Gupta S S, Parkash A, Ghangrekar A, Periasamy N and Sarma D D 2008 Bull. Mater. Sci. 31561

Nanda J, Kuruvilla B A and Sarma D D 1999 Phys. Rev. B59 7473

Orimi R L, Shahtahmasebi N, Tajabor N and Kompany A 2008 Physica E40 2894

Patil L A and Wani P A 2001 Cryst. Res. Technol. 36371

Sun L, Fu X, Wang M, Liu C, Liao C and Yan C $2000 \mathrm{~J}$. Lumin. 87-89 538

Sun Z P, Liu L, Zhang L and Jia D Z 2006 Nanotechnology 17 2266

Tell B, Damen T C and Porto S P S 1966 Phys. Rev. 144771

Vossmeyer T, Katsikas L, Giersig M, Popovic I G and Weller H 1994 J. Phys. Chem. 987665

Wang C, Tang K, Yang Q and Qian Y 2003 J. Electrochem. Soc. $150 \mathrm{G} 163$

Yu X B, Mao L H, Zhang F, Yang L Z and Yang S P 2004 Mater. Letts 583661

Zou B S, Little R B, Wang J P and EI-Sayed M A 1999 Int. J. Quantum Chem. 72439 